\title{
Eficácia do Herbicida Haloxyfop R (GR-142) Isolado e Associado ao 2,4-D no Controle de Híbridos de Milho RR $^{\circledR}$ Voluntário $^{1}$
}

\author{
Efficacy of Haloxyfop R (GR-142) herbicide isolated and associated to 2.4-D in \\ maize hybrids $\mathrm{RR}^{\circledR}$ volunteer control
}

Cleber Daniel de Goes Maciel ${ }^{2}$; Luiz Henrique Saes Zobiole ${ }^{3}$; João Igor de Souza ${ }^{4}$; Eige Hirooka ${ }^{5}$; Lucas Geaniny Noro Vargas de Lima ${ }^{5}$; Carlos Rafael Brandalise Soares ${ }^{5}$; Ricardo Angelo Deparis Pivatto ${ }^{5}$; Gabriel Martins Fuchs ${ }^{5}$; Enelise Osco Helvig ${ }^{5}$

Resumo - O milho voluntário Round up Ready ${ }^{\circledR}$ tem sido eficientemente manejado por graminicidas inibidores de ACCase, assim como na dessecação antes da semeadura da soja. Entretanto, antes da implantação da cultura, existem outras planta daninhas resistentes ao glyphosate como Conyza sp., Commelina sp., Ipomoea sp., Spermacoce latifolia and Richardia brasiliensis. Assim, a mistrua em tanque de inbidores de ACCase com 2,4-D esta aumentando nesse segmento. $\mathrm{O}$ milho $\mathrm{RR}^{\circledR}$ como planta voluntária na cultura da soja $\mathrm{RR}^{\circledR}$ tem sido manejado por herbicidas inibidores de ACCase tanto na dessecação de manejo como em pós-emergência. $\mathrm{O}$ presente trabalho avaliou a eficácia do herbicida Haloxyfop R (GF-142) isolado e associado ao 2,4-D (DMA $806 \mathrm{BR}^{\circledR}$ ) no controle de milho $\mathrm{RR}^{\circledR}$ voluntário em diferentes estádios. Um experimento foi conduzido a campo com os híbridos $\mathrm{RR}^{\circledR}$ DKB 240 PRO2, AG 8025 RR2, Pioneer 30F53HR e AG 9045RR2, utilizando o delineamento de blocos casualizados, em esquema fatorial $8 \times 2+1$, com quatro repetições. O primeiro fator representou haloxyfop GF-142 $\left(25,50\right.$ e $62 \mathrm{~g}$ i.a. ha $\left.{ }^{-1}\right)$ e clethodim (84 g i.a. ha ${ }^{-1}$ ) associados ou não ao herbicida 2,4-D (62 g e.a. $\mathrm{ha}^{-1}$ ). O segundo fator o estádio de desenvolvimento dos híbridos de milho, caracterizados por $\mathrm{V}_{5}$ e $\mathrm{V}_{7}$. O herbicida GR-142, isolados e/ou em mistura em tanque com 2,4-D, foi eficiente no controle em pós-emergência dos híbridos DKB 240 PRO2, AG 8025 RR2, AG 9045 RR2 e Pionner 30F 53HR, nos estádios $\mathrm{V}_{5} \mathrm{e} \mathrm{V}_{7}$. Os mesmo resultados foram observados para o padrão clethodim, isolado e/ou em mistura em tanque com 2,4-D. Entretanto, o controle no estádio $\mathrm{V}_{7}$ foi mais lento que no estádio $\mathrm{V}_{5}$.

Palavras-chaves: Inibidor de ACCase, mistura em tanque, plantas daninhas

Abstract - The volunteer corn Roundup Ready ${ }^{\circledR}$ has been effective managed by ACCase graminicides as burndown prior to sowing soybean. However, before crop installation, there are other glyphosate-tolerant weeds in the field such as Conyza sp., Commelina sp., Ipomoea sp.,

\footnotetext{
${ }^{1}$ Recebido para publicação em 08/04/2013 e aceito em 01/11/2013.

2 Professor, Dr. Universidade Estadual do Centro Oeste (UNICENTRO), Campus CEDETEG, Curso de Agronomia, R. Simeão Varela de Sá, 03, CEP: 85040-080, Guarapuava, PR, Brasil. Email: <cmaciel@ unicentro.br> (*autor para correspondência).

${ }^{3}$ Pesquisador Dr. Crop Protection, R\&D, Dow AgroSciences Industrial Ltda, Av. Nações Unidas, 14.171 - Sao Paulo, SP, Brasil.

${ }^{4}$ Mestrando do programa de pós-graduação em Agronomia da Universidade Estadual do Centro Oeste, Guarapuava, PR, Brasil.

${ }^{5}$ Acadêmicos do curso de Agronomia da Universidade Estadual do Centro Oeste, Guarapuava, PR, Brasil.
} 
Spermacoce latifolia and Richardia brasiliensis. Thus, the tank mixture of ACCase inhibitor herbicides with 2.4-D, is increasing in this segment. The present study evaluated the efficacy of Haloxyfop (GF-142) isolated and with 2.4-D (DMA $806 \mathrm{BR}^{\circledR}$ ) to volunteer corn control sprayed at different stages. One trial was conducted in field conditions with the corn $\mathrm{RR}^{\circledR}$ hybrids as DKB240PRO 2, AG8025RR2, Pioneer30F53HR and AG9045RR2 using a randomized block design in a split-plot factorial $8 \times 2+1$ with four replications. The main factor was the rate of haloxyfop GF-142 (25, 50 and $62 \mathrm{~g}$ a.i. $\left.\mathrm{ha}^{-1}\right)$ and clethodim $\left(84 \mathrm{~g}\right.$ a.i. ha $\left.{ }^{-1}\right)$ with or without the herbicide 2.4-D (670 $\mathrm{g}$ a.e. $\left.\mathrm{ha}^{-1}\right)$. The second factor was the application on volunteer corn stage at $\mathrm{V}_{5}$ and $\mathrm{V}_{7}$. The herbicide GR-142 at 25, 50 and $62 \mathrm{~g}$ a.i. ha ${ }^{-1}$, alone or with 2.4-D, was effective to control the volunteer corn plant hybrids DKB 240 PRO2, RR2 AG 8025, AG 9045 and RR2 Pioneer 30F53HR at both $\mathrm{V}_{5}$ and $\mathrm{V}_{7}$ stages. The same result was observed with the standard clethodim, alone and mixed with 2.4-D. However, the control at the $\mathrm{V}_{7}$ stage was slight slower than at the $\mathrm{V}_{5}$ stage.

Keywords: ACCase inhibitor, tank mix, weeds

\section{Introdução}

Como em qualquer outra cultura, plantas voluntárias de milho transgênicas, resistentes ao herbicida glyphosate, resultantes da germinação de grãos perdidos na colheita mecanizada são consideradas invasoras e podem assim como as plantas daninhas verdadeiras interferir na produtividade e qualidade de culturas em rotação/sucessão como a soja, também resistente ao glyphosate (Young \& Hart, 1997; Deen et al., 2006; Davis et al., 2008; Soltani et al., 2006; Marquardt et al., 2012).

A característica de tolerância ao glyphosate de híbridos de milho $\mathrm{RR}^{\circledR}$ (Roundup Ready ${ }^{\circledR}$ ) possibilita que esse único herbicida seja utilizado com seletividade à cultura, mas de forma contrária, impossibilita o controle quando altas populações do próprio milho RR® são plantas voluntárias, também conhecidas como tigueras, restevas e/ou guaxas, em culturas como a soja $\mathrm{RR}^{\circledR}$, por apresentarem resistência ao mesmo mecanismo de ação. Nesse sentido, Karan et al. (2013) mencionaram que a presença de cultivares resistentes como invasoras em culturas resistentes a herbicidas, como é o caso do milho voluntário $\mathrm{RR}^{\circledR}$, pode se tornar plantas de difícil controle, uma vez que as alternativas para o manejo químico ficam limitadas na

dessecação de manejo de plantio direto, tanto em pré-semeadura como em pós-colheita.

Em sistemas de rotação/sucessão em que o milho $\mathrm{RR}^{\circledR}$ aparece como planta voluntária, a aplicação combinada de glyphosate com graminicidas inibidores da enzima ACCase constituem uma opção tanto para dessecação de manejo antecedendo a semeadura direta, como no controle em pósemergência após a instalação da cultura da soja $\mathrm{RR}^{\circledR}$. Esta opção de manejo também vem sendo utilizada com sucesso em áreas onde há ocorrência de plantas daninhas resistentes ao glyphosate, como azevém (Lolium multiflorum) e capim-amargoso (Digitaria insularis). Entretanto, em condições onde o complexo da infestação ainda é constituído por biótipos de buva (Conyza sp.) resistentes e outras espécies tolerantes ao glyphosate, como trapoeraba (Commelina sp.), corda-de-viola (Ipomoea sp.), amendoim-bravo (Euphorbia heterophylla), erva-quente (Spermacoce latifolia) e poaiabranca (Richardia brasiliensis), a operação de manejo em pré-semeadura ou pós-colheita tem sido realizada por associações de glyphosate com inibidores da ACCase e 2,4-D, visando ampliar a eficiência do espectro de ação.

O haloxyfop-methyl é um herbicida sistêmico, do grupo químico ariloxifenoxipropiônicos (APP), que inibe a enzima acetilcoenzima-A carboxilase 
(ACCase), responsável pela catalização das reações de síntese de ácidos graxos, interrompendo a formação dos lipídios e membranas celulares (Vidal \& Merotto Jr, 2001; Roman, et al. 2007). No Brasil é registrado para o controle de gramíneas em pós-emergência nas culturas da soja, algodão e feijão, mas tem restrição de não utilizar em mistura em tanque com produtos à base de 2,4D (Rodrigues \& Almeida, 2011).

$\mathrm{Na}$ literatura encontram-se relatos do efeito antagônico do 2,4-D sobre a ação de graminicidas inibidores da ACCase, sendo esses relacionados a redução da translocação e aumento do metabolismo dos herbicidas do grupo dos ariloxifenoxipropiônicos. (Roman, et al. 2007; Trezzi et al. 2007). Entretanto, ainda são restritos as informações que consideram a ação negativa do 2,4-D quando associado com o glyphosate e inibidores da ACCase em uma mesmas mistura em tanque, tanto no controle de voluntárias, como é no caso do milho $\mathrm{RR}^{\circledR}$, assim como de outras espécies de plantas daninhas resistentes e/ou tolerantes aos herbicidas.

O objetivo deste trabalho foi avaliar a eficácia do herbicida haloxyfop (GF-142) isolado e associado ao 2,4-D (DMA $806 \mathrm{BR}^{\circledR}$ ) no controle de híbridos de milho $\mathrm{RR}^{\circledR}$ voluntários, em diferentes estádios de desenvolvimento.

\section{Material e Métodos}

O ensaio foi realizado no Campo Experimental do Departamento de Agronomia da Universidade Estadual do Centro-Oeste UNICENTRO/DEAGRO, no Campus CEDETEG de Guarapuava/PR. A área experimental foi implantada nas coordenadas

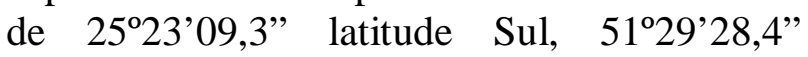
longitude Oeste e $984 \mathrm{~m}$ de altitude. O clima da região, segundo classificação de KöeppenGeiger, é do tipo subtropical Cfb (Peel et al., 2007), ou seja, com chuvas bem distribuídas durante o ano e verões amenos. Os dados de climatológicos ocorridos durante o período experimental encontram-se descritos na Tabela 1, os quais foram obtidos na estação meteorológica do Instituto Agronômico do Paraná (IAP), localizada dentro do Campus universitário, e que se encontrava a $500 \mathrm{~m}$ do experimento.

Tabela 1. Temperatura $\left({ }^{\circ} \mathrm{C}\right.$, máxima e mínima), umidade relativa do ar (\% média) e precipitações $(\mathrm{mm})$ semanais observadas durante o período de condução do experimento para o controle de híbridos de milho $\mathrm{RR}^{\circledR}$ voluntário, onde as aplicações foram realizadas em 20/12/2012. Guarapuava - PR, 2012/2013.

\begin{tabular}{|c|c|c|c|c|c|c|c|c|c|c|c|c|}
\hline \multirow{3}{*}{ Semanas } & \multicolumn{4}{|c|}{ Dezembro 2012 } & \multicolumn{4}{|c|}{ Janeiro 2013 } & \multicolumn{4}{|c|}{ Fevereiro 2013 } \\
\hline & \multicolumn{2}{|c|}{ Temp. } & \multirow{2}{*}{$\begin{array}{l}\text { Umid } \\
\text { Méd }\end{array}$} & \multirow{2}{*}{$\begin{array}{l}\text { Chuva } \\
\text { mm }\end{array}$} & \multicolumn{2}{|c|}{ Temp. } & \multirow{2}{*}{$\begin{array}{l}\text { Umid } \\
\text { Méd }\end{array}$} & \multirow{2}{*}{$\begin{array}{c}\text { Chuva } \\
\text { mm }\end{array}$} & \multicolumn{2}{|c|}{ Temp. } & \multirow{2}{*}{$\begin{array}{l}\text { Umid } \\
\text { Méd }\end{array}$} & \multirow{2}{*}{$\begin{array}{c}\text { Chuva } \\
\text { mm }\end{array}$} \\
\hline & Máx & Mín & & & Máx & Mín & & & Máx & Mín & & \\
\hline $1^{a} S$ & 28,2 & 17,3 & 72,1 & 4,8 & 24,5 & 16,3 & 82,8 & 55,8 & 25,9 & 16,1 & 72,5 & 85,9 \\
\hline $2^{\mathrm{a}} \mathrm{Ser}$ & 27,8 & & 78,2 & 72,6 & 25,5 & 5,2 & 69,1 & 60,2 & 23,5 & 17,3 & 85,3 & 42,5 \\
\hline $3^{\text {a }}$ Semana & 28,2 & 18,5 & 74,1 & 30,4 & 25,6 & 15,4 & 68,4 & 3,0 & 27,3 & 18,0 & 80,8 & 104,2 \\
\hline $4^{a}$ Semana & 28,0 & 18,2 & 76,6 & 19,0 & 27,7 & 15,0 & 63,7 & 0,0 & 27,1 & 16,3 & 73,8 & 39,2 \\
\hline $5^{\mathrm{a}}$ Semana $($ dias 29,30 e 31$)$ & 24,7 & 18,1 & 86,5 & 51,8 & 28,6 & 15,9 & 58,3 & 0,0 & & & & \\
\hline Média*/Soma** & $27,4^{*}$ & $18,0^{*}$ & $77,5^{*}$ & $178,6^{* * *}$ & $26,4^{*}$ & $15,6^{*}$ & $68,5^{*}$ & $119,0^{\text {*** }}$ & $25,9^{*}$ & $16,9^{*}$ & $78,1^{*}$ & $185,9^{* * *}$ \\
\hline
\end{tabular}

Temp. $=$ Temperatura $\left({ }^{\circ} \mathrm{C}\right) ;$ Umid $=$ Umidade do ar $(\%)$; Chuva = precipitação $(\mathrm{mm})$; Máx. = Máxima; Mín. = Mínima; Méd. = Media. Fonte: Estação Meteorológica IAP/UNICENTRO/CEDETEG - CEP 85040-080. Guarapuava - PR.

O solo é classificado como Latossolo Bruno ácrico típico (EMBRAPA, 2006), de textura muito argilosa (50\% de argila, $20 \%$ de areia e $30 \%$ de silte), o qual a análise química, em amostras de 0 a $20 \mathrm{~cm}$ de profundidade, revelou $\mathrm{pH}$ em $\mathrm{CaCl}$ de 4,8; 6,51 $\mathrm{cmol}_{\mathrm{c}} \mathrm{dm}^{-3} \mathrm{de}$ $\mathrm{H}^{+}+\mathrm{Al}^{3+} ; 4,10 \mathrm{cmol}_{\mathrm{c}} \mathrm{dm}^{-3} \mathrm{de} \mathrm{Ca}^{+2} ; 2,3$ mmol $_{\mathrm{c}}$ $\mathrm{dm}^{-3}$ de $\mathrm{Mg}^{+2} ; 0,35 \mathrm{cmol}_{\mathrm{c}} \mathrm{dm}^{-3}$ de $\mathrm{K}^{+} ; 1,8 \mathrm{mg}$ $\mathrm{dm}^{-3}$ de $\mathrm{P}$ (Mehlich) e 3,09 $\mathrm{g} \mathrm{dm}^{-3}$ de C, respectivamente. 
Os quatro híbridos de milho $\mathrm{RR}^{\circledR} \mathrm{DKB}$ 240 PRO 2, AG 8025 RR2, Pioneer 30F 53HR e AG 9045 RR2 foram estudados utilizando o delineamento experimental de blocos casualizados, em esquema fatorial $8 \times 2+1$, com quatro repetições. $\mathrm{O}$ primeiro fator representou os tratamentos com os herbicidas GF-142 [haloxyfop-methyl (R) $\left(25,50\right.$ e 62 g i.a. ha $\left.{ }^{-1}\right)$ ] e Select ${ }^{\circledR}$ [clethodim $\left(84\right.$ g i.a. ha $\left.{ }^{-1}\right)$ ] associados ou não ao herbicida DMA 86 BR $^{\circledR}[2,4-\mathrm{D}(62 \mathrm{~g}$ e.a. $\left.\left.h^{-1}\right)\right]$, e o segundo fator o estádio de desenvolvimento dos híbridos de milho, caracterizados por $\mathrm{V}_{5}$ e $\mathrm{V}_{7}$ (cinco e sete folhas totalmente expandidas) (Figuras 1 e 2). As unidades experimentais foram constituídas por parcelas de 4,0 × 5,0 m, assim como todos os tratamentos e respectivas dosagens utilizadas no experimento encontram-se na Tabela 2.

Tabela 2. Tratamentos e respectivas dosagens utilizadas no experimento para o controle de híbridos de milho RR voluntário. Guarapuava - PR, 2012/2013.

\begin{tabular}{|c|c|c|c|c|}
\hline \multirow[b]{2}{*}{ Tratamentos } & \multirow{2}{*}{$\begin{array}{l}\text { Dosagem } \\
\text { g i.a. ha } \\
\text { ou } \\
\text { g e.a. ha } \\
\text { he }\end{array}$} & \multirow[b]{2}{*}{$\begin{array}{l}\text { Modo de } \\
\text { aplicação }^{\underline{1}}\end{array}$} & \multicolumn{2}{|c|}{ Formulação comercial } \\
\hline & & & $\begin{array}{c}\text { Marca, tipo de formulação } \\
\text { e concentração }\end{array}$ & $\begin{array}{l}\text { Dosagem } \\
\text { p.c. } \text { ha }^{-1}\end{array}$ \\
\hline $\begin{array}{l}1 \text { haloxyfop-methyl }{ }^{3 /} \\
\text { haloxyfop-methyl }\end{array}$ & $\begin{array}{l}25,0 \\
25,0\end{array}$ & $\begin{array}{l}\mathrm{PÓS}^{(\mathrm{V} 5)} \\
\mathrm{PÓS}^{(\mathrm{V} 7)}\end{array}$ & $\begin{array}{l}\mathrm{GF}-142, \mathrm{EC}^{-2},[539,98] \mathrm{g} / \mathrm{L} \\
\mathrm{GF}-142, \mathrm{EC}^{-2},[539,98] \mathrm{g} / \mathrm{L}\end{array}$ & $\begin{array}{l}0,0463 \mathrm{~L} \\
0,0463 \mathrm{~L}\end{array}$ \\
\hline $\begin{array}{l}2 \text { haloxyfop-methyl }+2,4-\mathrm{D}^{3 /} \\
\text { haloxyfop-methyl }+2,4-\mathrm{D}^{\underline{3} /}\end{array}$ & $\begin{array}{l}25,0+670 \\
25,0+670\end{array}$ & $\begin{array}{l}\text { PÓS }^{(\mathrm{V} 5)} \\
\text { PÓS }^{(\mathrm{V} 7)}\end{array}$ & 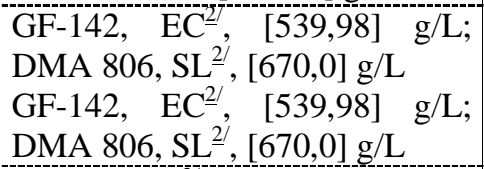 & $0,0463+1,0 \mathrm{~L}$ \\
\hline $\begin{array}{l}3 \text { haloxyfop-methyl }{ }^{3 /} \\
\text { haloxyfop-methyl }{ }^{3 /}\end{array}$ & $\begin{array}{l}50,0 \\
50,0\end{array}$ & $\begin{array}{l}\mathrm{PÓS}^{(\mathrm{V5})} \\
\mathrm{PÓS}^{(\mathrm{V7})}\end{array}$ & $\begin{array}{l}\mathrm{GF}-142, \mathrm{EC}^{2},[539,98] \mathrm{g} / \mathrm{L} \\
\mathrm{GF}-142, \mathrm{EC}^{2},[539,98] \mathrm{g} / \mathrm{L}\end{array}$ & $\begin{array}{l}0,0926 \mathrm{~L} \\
0,0926 \mathrm{~L}\end{array}$ \\
\hline $\begin{array}{l}4 \text { haloxyfop-methyl }+2,4-\mathrm{D}^{3 /} \\
\text { haloxyfop-methyl }+2,4-\mathrm{D}^{\underline{3} /}\end{array}$ & $\begin{array}{l}50,0+670 \\
50,0+670\end{array}$ & $\begin{array}{l}\text { PÓS } \\
\text { PÓS }^{(\mathrm{V} 7)}\end{array}$ & 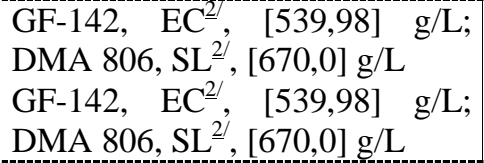 & $\begin{array}{l}0,0926+1,0 \mathrm{~L} \\
0,0926+1,0 \mathrm{~L}\end{array}$ \\
\hline $\begin{array}{l}5 \text { haloxyfop-methyl }{ }^{3 /} \\
\text { haloxyfop-methyl }{ }^{3 /}\end{array}$ & $\begin{array}{l}62,0 \\
62,0\end{array}$ & $\begin{array}{l}\text { PÓS }^{(\sqrt{ } 5)} \\
\text { PÓS }^{(V 7)}\end{array}$ & $\begin{array}{l}\mathrm{GF}-142, \mathrm{EC}^{2}-[539,98] \mathrm{g} / \mathrm{L} \\
\mathrm{GF}-142, \mathrm{EC}^{2}{ }^{2},[539,98] \mathrm{g} / \mathrm{L}\end{array}$ & $\begin{array}{l}0,115 \mathrm{~L} \\
0,115 \mathrm{~L}\end{array}$ \\
\hline $\begin{array}{l}6 \text { haloxyfop-methyl }+2,4-\mathrm{D}^{3 /} \\
\text { haloxyfop-methyl }+2,4-\mathrm{D}^{3 /}\end{array}$ & $\begin{array}{l}62,0+670 \\
62,0+670\end{array}$ & $\begin{array}{l}\text { PÓS }^{(\mathrm{V} 5)} \\
\text { PÓS }^{(\mathrm{V} 7)}\end{array}$ & 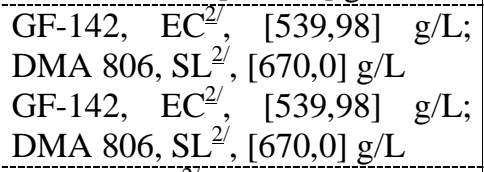 & $0,115+1,0 \mathrm{~L}$ \\
\hline $\begin{array}{l}7 \text { clethodim }^{4}{ }^{-1} \\
\text { clethodim }\end{array}$ & $\begin{array}{l}84,0 \\
84,0\end{array}$ & $\begin{array}{l}\text { PÓS }^{(\sqrt{ } 5)^{-1}} \\
\text { PÓS }^{(\mathrm{V} 7)}\end{array}$ & $\begin{array}{l}\mathrm{GF}-142, \mathrm{EC}^{2}{ }^{2},[240,0] \mathrm{g} / \mathrm{L} \\
\mathrm{GF}-142, \mathrm{EC}^{2},[240,0] \mathrm{g} / \mathrm{L}\end{array}$ & $\begin{array}{l}0,350 \mathrm{~L} \\
0,350 \mathrm{~L}\end{array}$ \\
\hline $\begin{array}{l}8 \text { clethodim }+2,4-\mathrm{D}^{4 \prime} \\
\text { clethodim }+2,4-\mathrm{D}^{4 \prime}\end{array}$ & $\begin{array}{l}84,0+670 \\
84,0+670\end{array}$ & $\begin{array}{l}\text { PÓS } \\
\text { PÓS }^{(\mathrm{V} 5)}\end{array}$ & 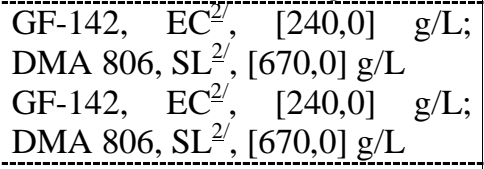 & $0,350+1,0 \mathrm{~L}$ \\
\hline 9 Testemunha sem herbicida & - & - & - & - \\
\hline
\end{tabular}

I'PÓS: Pós-emergência em estádio de desenvolvimento de 5 ou 7 folhas total expandidas $\left(\mathrm{V}_{5}\right.$ ou $\left.\mathrm{V}_{7}\right)$. ${ }^{2 /}$ EC: Concentrado emulsionável; SL: Concentrado solúvel; ${ }^{3 /}$ Utilizou-se $1,0 \mathrm{~L} \mathrm{pc} \mathrm{ha}{ }^{-1}$ de Joint Oil ${ }^{\circledast}$ (Adjuvante do grupo dos hidrocarbonetos alifáticos); ${ }^{4 /}$ Utilizou-se $1,0 \mathrm{~L} \mathrm{pc} \mathrm{ha}{ }^{-1} \mathrm{de}$ Lanzar $^{\circledR}$ (Adjuvante do grupo dos alquil ester etoxilado do ácido fosfórico).

A semeadura dos híbridos de milho foi realizada em 23/11/2012 e 07/12/2012, visando a simulação de plantas voluntárias em dois estádios de desenvolvimento no momento da aplicação dos tratamentos herbicidas. A profundidade de semeadura dos híbridos foi entre 3 a $5 \mathrm{~cm}$, em solo previamente adubado com $100 \mathrm{~kg} \mathrm{ha}^{-1}$ do formulado 8-20-15 (NPK).

As aplicações dos tratamentos foram realizadas em 20/12/2012 utilizando-se um pulverizador costal pressurizado a $\mathrm{CO}_{2}$, equipado com seis pontas TTi 110.15 
(fabricante Teejet ${ }^{\circledR}$ ), espaçadas entre si em 0,5 $\mathrm{m}$ e a $0,5 \mathrm{~m}$ de altura das parte aérea das plantas, constituindo taxa de aplicação de 150 $\mathrm{L} \mathrm{ha}^{-1}$. O início e final da aplicação dos tratamentos, realizada no período da manhã, no horário de $18 \mathrm{~h} 10 \mathrm{~m}$ a $18 \mathrm{~h} 45 \mathrm{~m}$, quando a média da umidade relativa do ar, temperatura e velocidade dos ventos foram, respectivamente, de 68,7 a $70,3 \% ; 23,4$ a $21,5{ }^{\circ} \mathrm{C}$ e com ventos de 0,3 a $0,6 \mathrm{~km} \mathrm{~h}^{-1}$. No momento da aplicação, os estádios fenológicos das plantas de milho foram caracterizados por $\mathrm{V}_{5}$ e $\mathrm{V}_{7}$ (cinco a sete folhas completamente expandidas), e as densidades de plantas variando de 48 a 69 plantas $\mathrm{m}^{-2}$.

Para o controle preventivo da lagartado-cartucho, aos 10 dias antes da aplicação dos herbicidas, efetuou-se ainda uma aplicação complementar do inseticida Lannate $^{\circledR}$, na dosagem de $600 \mathrm{~mL} \mathrm{pc} \mathrm{ha}{ }^{-1}$.

A porcentagem de controle do milho voluntário foi estabelecida por meio de escala de notas visuais (SBCPD, 1995), onde 0\% correspondeu à ausência de injúrias e $100 \%$ à morte das plantas aos 7, 14, 21, 28 e 42 DAA (dias após aplicação) dos tratamentos.

Os dados foram submetidos à análise de variância pelo teste $\mathrm{F}$ e suas médias comparadas pelo teste tukey, a $5 \%$ de probabilidade.

\section{Resultados e Discussão}

Para o controle do híbrido de milho DKB 240 PRO2 (Tabela 3), observa-se que apenas a menor dose de GR-142 (25 $\left.\mathrm{g} \mathrm{ha}^{-1}\right) \mathrm{em}$ mistura em tanque com o DMA $806 \mathrm{BR}^{\circledR}(670$ $\mathrm{g} \mathrm{ha}^{-1}$ ) causou os maiores efeitos antagônico aos 7 e 14 DAA, para os dois estádios de desenvolvimento $\left(\mathrm{V}_{5} \mathrm{e}_{7}\right)$. Quando utilizado $25 \mathrm{~g} \mathrm{ha}^{-1}$ de GR-142 de forma isolada, constatou-se níveis de controle próximos ao satisfatório e satisfatório aos 7 DAA, respectivamente, para os estádios $V_{5}$ e $V_{7}$ (73,8\% e $81,5 \%)$, os quais ambos evoluíram para controle eficiente $(92,8 \%$ e $90,3 \%)$ aos 14 DAA (Figura 1). Esse tratamento não diferiu significativamente da excelente ação das maiores doses de GR-142 e do tratamento Select ${ }^{\circledR}\left(84 \mathrm{~g} \mathrm{ha}^{-1}\right)$, mesmo quando em mistura com DMA $806 \mathrm{BR}^{\circledR}\left(670 \mathrm{ha}^{-1}\right)$. A partir dos 21 DAA, a menor dose do GR-142 (25 $\left.\mathrm{g} \mathrm{ha}^{-1}\right)$, isolada ou em mistura com DMA $806 \mathrm{BR}^{\circledR}$, apresentaram excelentes níveis de controle do híbrido DKB 240 PRO2, principalmente em estádio $V_{5}$, não diferindo significativamente das maiores doses de GR-142, assim como do Select ${ }^{\circledR}\left(84 \mathrm{~g} \mathrm{ha}^{-1}\right)$ e Select $^{\circledR}+$ DMA 806 BR $^{\circledR}$ $\left(84+670 \mathrm{~g} \mathrm{ha}^{-1}\right)$. Para o estádio $\mathrm{V}_{7}$, observa-se que a menor dose de GR-142 (25 $\left.\mathrm{g} \mathrm{ha}^{-1}\right)$, isolada ou em mistura com DMA $806 \mathrm{BR}^{\circledR}$, apresentou controle altamente eficiente até os 42 DAA ( $\leq 92,8 \%)$, mas ainda assim na maioria das vezes foi significativamente inferior as doses de 50 e 62,0 $\mathrm{g} \mathrm{ha}^{-1}$ de GR142.

Apesar de 25 e $50 \mathrm{~g} \mathrm{ha}^{-1}$ de GR-142 aos 7 e 14 DAA terem apresentado maior antagonismo para $\mathrm{o}$ estádio $\mathrm{V}_{7}$ quando utilizado em mistura com DMA $806 \mathrm{BR}^{\circledR}$, foi possível constatar que o tratamento com $62 \mathrm{~g}$ $\mathrm{ha}^{-1}$ de GR-142, teve comportamento semelhante aos padrões Select ${ }^{\circledR}$ e Select $^{\circledR}+$ DMA 806 BR $^{\circledR}$, em ambos estádios de desenvolvimento. A partir dos 14 DAA, as doses de 50 e $62 \mathrm{~g} \mathrm{ha}^{-1}$ de GR-142, isolada ou em mistura com DMA 806 BR $^{\circledR}$, apresentaram elevada eficiência no controle do híbrido DKB 240 PRO2, não diferindo significativamente dos padrões Select ${ }^{\circledR}$ e Select $^{\circledR}+$ DMA 806 BR $^{\circledR}$ (Figura 2). 
Tabela 3. Porcentagens de controle de milho RR híbrido voluntário DKB 240 PRO 2 aos 7, 14, 21, 28 e 42 dias após aplicação (DAA) em pós-emergência do herbicida GF-142 [haloxyfopmethyl (R)], em estádios de desenvolvimento $V_{5}$ e V $V_{7}$. Guarapuava - PR, 2012/2013.

\begin{tabular}{|c|c|c|c|c|c|c|}
\hline \multirow{3}{*}{ Tratamentos/Estádios } & \multirow{3}{*}{$\begin{array}{c}\text { Dosagem } \\
\text { g i.a. ou } \\
\text { e.a ha-1 }\end{array}$} & \multicolumn{5}{|c|}{$\%$ de controle milho voluntário DKB 240 PRO 2} \\
\hline & & 7 DAA & 14 DAA & 21 DAA & 28 DAA & 42 DAA \\
\hline & & $\begin{array}{ll}\mathrm{V}_{5} & \mathrm{~V}_{7} \\
\end{array}$ & $\begin{array}{ll}\mathrm{V}_{5} & \mathrm{~V}_{7} \\
\end{array}$ & $\mathrm{~V}_{5}$ & $\mathrm{~V}_{7}$ & $\mathbf{V}_{5}$ \\
\hline 1. GR-142 & 25 & 73,8 bB 81,5 bc $A$ & 92,8 aA 90,3 aA & 98,3 aA 93,5 bB & 98,5 aA 94,5 bB & 99,3 aA 97,8 bcB \\
\hline 2. GR-142 + DMA $806 ?$ & $25+670$ & 66,3 bA $40,0 \quad \mathrm{~dB}$ & 80,8 bA $\quad 55,8$ bB & 97,8 aA 92,8 bB & 96,0 aA 95,8 ab & 98,5 aA $97,3 \mathrm{cA}$ \\
\hline 3. GR-142? & 50 & 97,3 aA 92,8 aA & 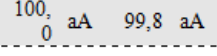 & 100,0 aA 99,8 aA & 100,0 aA 99,0 aA & 100,0 aA 99,8 abA \\
\hline 4. GR-142+DMA 806? & $50+670$ & 91,3 aA 75,0 cB & 98,5 aA 91,5 aA & 100,0 aA 96,8 ab & 100,0 aA 96,5 abB & 100,0 aA 99,3 abcA \\
\hline 5. GR-142 & 62 & 98,5 aA 91,5 abB & 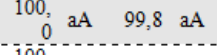 & 100,0 aA 99,8 aA & 100,0 aA 99,0 aA & 100,0 aA 99,8 abA \\
\hline 6. GR-142 + DMA $806 ?$ & $62+670$ & 98,0 aA 82,0 abcB & 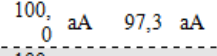 & 100,0 aA 99,5 aA & 100,0 aA 99,5 aA & 100,0 aA 100,0 aA \\
\hline 7. Select ${ }^{\frac{3}{2}}$ & 84 & 99,5 aA 91,0 abB & 0 aA 96,5 aA & 100,0 aA 98,8 aA & 100,0 aA 97,8 ab & 100,0 aA 99,8 abA \\
\hline 8. Select + DMA 806 ' & $84+670$ & 99,0 aA 87,8 abB & $\begin{array}{r}100, \\
0\end{array}$ & 100,0 aA $96,3 \stackrel{\mathrm{ab}}{\mathrm{B}}$ & 100,0 aA $98,0 \stackrel{\mathrm{ab}}{\mathrm{A}}$ & 100,0 aA 99,5 abA \\
\hline 9. Testemunha sem aplicação & - & $0,0 \quad \mathrm{c} \quad 0,0 \quad \mathrm{e}$ & $0,0 \mathrm{c} \quad 0,0 \mathrm{c}$ & $0,0 \quad \mathrm{~b} \quad 0,0 \mathrm{c}$ & $0,0 \mathrm{~b} \quad 0,0 \mathrm{c}$ & $0,0 \quad b \quad 0,0 \quad d$ \\
\hline Frratamento & & $359,73^{*}$ & $338,47^{*}$ & $649,851^{*}$ & $2315,85^{*}$ & $9564,34 *$ \\
\hline F $_{\text {Estádio }}$ & & $67,67^{*}$ & $21,82^{*}$ & $23,99^{*}$ & $12,55^{*}$ & $5,45^{*}$ \\
\hline Frratamentox Estádio & & $8,95^{*}$ & $4,93^{*}$ & $2,54^{*}$ & $1,09^{\mathrm{NS}}$ & $0,63^{\mathrm{NS}}$ \\
\hline CV $(\%)$ & & 6,20 & 6,05 & 2,09 & 2,21 & 1,08 \\
\hline DMS ${ }_{\text {Tratamento }}$ & & 10,76 & 11,50 & 4,19 & 4,42 & 2,19 \\
\hline DMSEstádio & & 6,67 & 7,12 & $2,60^{-}$ & $2,74^{--}$ & $1, \overline{3} \overline{6}^{-}$ \\
\hline
\end{tabular}

${ }^{1 /} \mathrm{EF}=$ Estádio de desenvolvimento das plantas de milho com 5 ou 7 folhas totalmente expandidas (V5 ou V7); ${ }^{2 /}$ Utilizou-se $1,0 \mathrm{~L}$ pc ha ${ }^{-1}$ de Joint $\mathrm{Oil}^{\circledR}$ (Adjuvante do grupo dos hidrocarbonetos alifáticos); ${ }^{3 /}$ Utilizou-se $1,0 \mathrm{~L} \mathrm{pc} \mathrm{ha}^{-1}$ de Lanzar $^{\circledR}$ (Adjuvante do grupo dos alquil ester etoxilado do ácido fosfórico); - DAA = dias após aplicação; - Médias na mesma coluna seguidas da mesma letra não diferem entre si, a 5\% de probabilidade pelo teste de Tukey. ${ }^{*} \mathrm{p} \leq 0,05{ }^{\mathrm{NS}}=$ não significativo.

Para o controle do híbrido de milho AG 8025 RR2 (Tabela 4), observa-se que houve grande similaridade dos resultados obtidos com o híbrido DKB 240 PRO2, sendo que novamente apenas aos 7 e 14 DAA a menor dose do GR-142 (25 $\left.\mathrm{g} \mathrm{ha}^{-1}\right)$ em mistura com o DMA $806 \mathrm{BR}^{\circledR}$ promoveu efeito antagônico principalmente para o estádio $\mathrm{V}_{7}$. Aos 7 DAA, as doses de 50 e $62 \mathrm{~g} \mathrm{ha}^{-1}$ de GR-142 também apresentaram inicialmente influência negativa quando em mistura com DMA $806 \mathrm{BR}^{\circledR}$ apenas para o estádio $\mathrm{V}_{7}$, uma vez que os níveis de controle foram insatisfatórios $(76,5 \%$ e $78,3 \%)$ e significativamente inferiores ao padrão Select ${ }^{\circledR}$ e Select ${ }^{\circledR}$ + DMA 806 BR $^{\circledR}$. Entretanto, a partir dos 14 DAA, para 50 e $62 \mathrm{~g} \mathrm{ha}^{-1} \mathrm{de}$ GR-142 e dos 21 DAA para $25 \mathrm{~g} \mathrm{ha}^{-1}$ de GR142, isolados ou em mistura com DMA 806 $\mathrm{BR}^{\circledR}$, os níveis de controle do híbrido AG 8025 RR2 foram altamente eficientes $(\leq 95,3 \%)$, não diferindo significativamente dos tratamentos Select ${ }^{\circledR}$ e Select $^{\circledR}$ + DMA 806 BR $^{\circledR}$ (Figura 2).
Para o estádio $\mathrm{V}_{5}$, as doses de 50 e $62 \mathrm{~g} \mathrm{ha}^{-1}$ de GR-142, isoladas ou em mistura com DMA $806 \mathrm{BR}^{\circledR}$, foram altamente eficientes a partir dos 7 DAA, sendo ainda que aos 21 DAA atingiram eficiência máxima (100\%).

Para o controle dos híbridos de milho Pionner 30F 53HR e AG 9045 RR2 (Tabelas 5 e 6), as mesmas observações feitas para os híbridos DKB 240 PRO2 e AG 8025 RR2 são válidas, uma vez que novamente aos 7 DAA o GR-142 a dose de $25 \mathrm{~g} \mathrm{ha}^{-1}$ em mistura com DMA 806 BR $^{\circledR}$ causaram antagonismo, principalmente para o estádio $\mathrm{V}_{7}$. De forma geral, para o estádio $\mathrm{V}_{7}$ as doses de 50 e $62 \mathrm{~g}$ ha $^{-1}$ de GR-142 aos 7 DAA também apresentaram antagonismo quando em mistura com DMA 806 BR $^{\circledR}$, constituindo níveis de controle insatisfatórios para o híbrido Pionner 30F 53HR $(77,0 \%$ e $78,8 \%)$ e apenas satisfatório para AG 9045 RR2 $(82,0 \%$ e $82,3 \%$ ), sendo ambos significativamente inferiores ao Select $^{\circledR}$ e Select ${ }^{\circledR}+$ DMA 806 $\mathrm{BR}^{\circledR}$. 

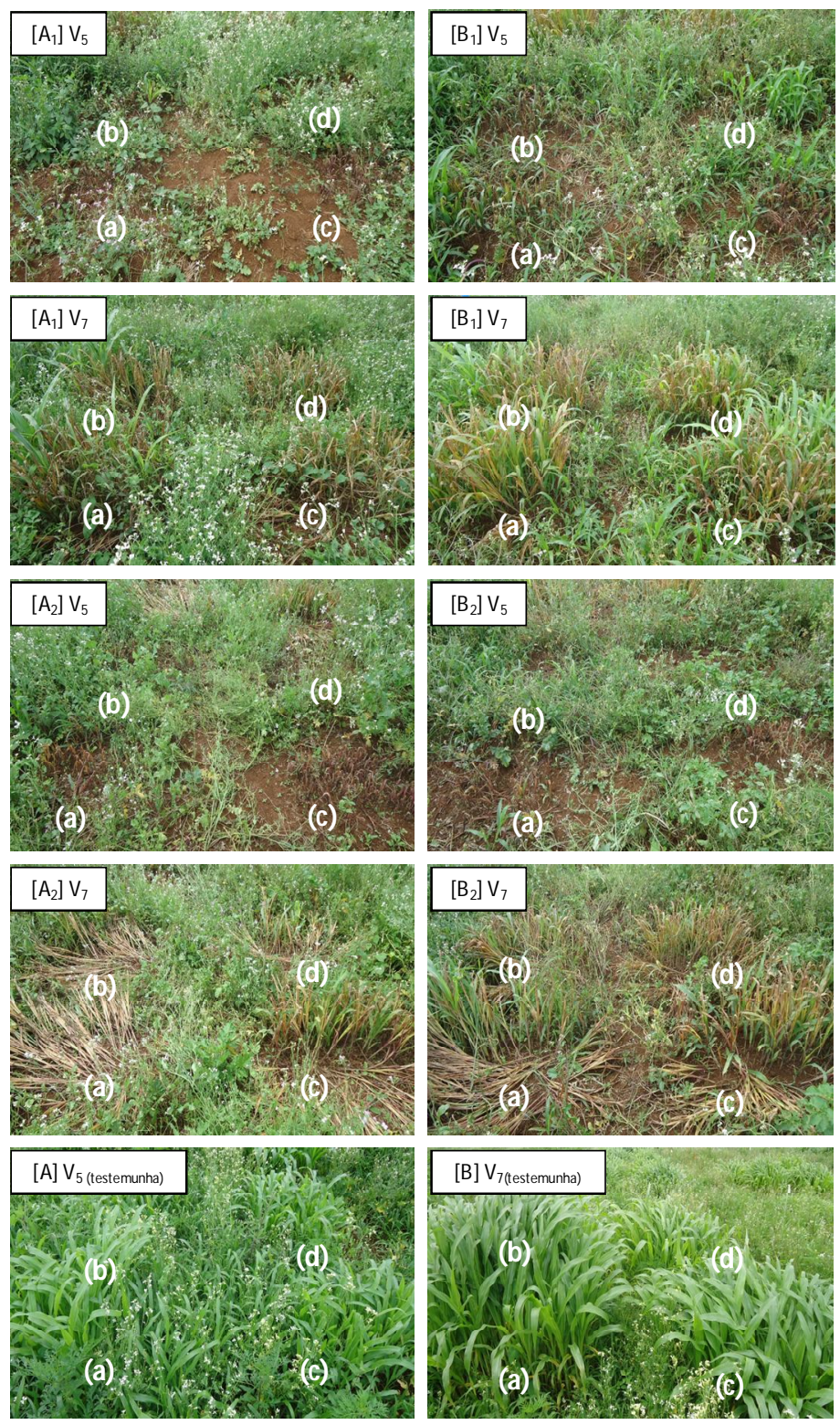

Figura 1. Ilustração do controle de milho RR voluntário DKB 240 PRO 2 (a), AG 8025 RR2 (b), Pioneer 30F 53HR (c) e AG 9045 RR2 (d) aos 14 dias após aplicação (DAA) em pós-emergência dos tratamentos herbicida GF-142 (25 $\left.\mathrm{g} \mathrm{ha}^{-1}\right)\left[\mathrm{A}_{1}\right]$, GF-142 + DMA $806 \mathrm{BR}^{\circledR}\left(25+670 \mathrm{~g} \mathrm{ha}^{-1}\right)$ $\left[\mathrm{B}_{1}\right], \mathrm{GF}-142\left(50 \mathrm{~g} \mathrm{ha}^{-1}\right)\left[\mathrm{A}_{2}\right], \mathrm{GF}-142+\mathrm{DMA} 806 \mathrm{BR}^{\circledR}\left(50+670 \mathrm{~g} \mathrm{ha}^{-1}\right)\left[\mathrm{B}_{2}\right]$ e testemunhas em estádios $\mathrm{V}_{5}$ e $\mathrm{V}_{7}$. Guarapuava - PR, 2012/2013.

Aos 14 DAA, também foi possível detectar antagonismo no controle dos híbridos Pionner 30F 53HR e AG 9045 RR2 para mistura de GR-142 + DMA 806 BR $^{\circledR}$, nas doses de $50+670 \mathrm{~g} \mathrm{ha}^{-1}$, sendo este efeito mais expressivo que o ocorrido com os híbridos DKB 240 PRO2 e AG 8025 RR2 (Figura 1). É importante ressaltar que comparativamente o híbrido Pionner 30F 53HR foi o mais tolerante a ação da menor dose de GR-142 (25 $\left.\mathrm{g} \mathrm{ha}^{-1}\right)$, quando utilizado isolado ou em mistura com DMA 806 BR $^{\circledR}$, uma vez que apesar de pequenas as diferenças, o mesmo apresentou os menores níveis de controle até 21 e/ou 28 DAA. 
Tabela 4. Porcentagens de controle de milho RR híbrido voluntário AG 8025 RR2 aos 7, 14, 21, 28 e 42 dias após aplicação (DAA) em pós-emergência do herbicida GF-142 [haloxyfop-methyl (R)], em estádios de desenvolvimento $\mathrm{V}_{5}$ e $\mathrm{V}_{7}$. Guarapuava - PR, 2012/2013.

\begin{tabular}{|c|c|c|c|c|c|c|}
\hline \multirow{3}{*}{ Tratamentos/Estádios } & \multirow{3}{*}{$\begin{array}{c}\text { Dosagem } \\
\text { g i.a. ou } \\
\text { e.a ha-1 }\end{array}$} & \multicolumn{5}{|c|}{ \% de controle milho voluntário AG 8025 RR2 } \\
\hline & & 7 DAA & 14 DAA & 21 DAA & 28 DAA & 42 DAA \\
\hline & & $\mathrm{V}_{7}$ & $\mathrm{~V}_{7}$ & $\mathrm{~V}_{7}$ & $V_{5}$ & $V_{5}$ \\
\hline 1. GR-142 & 25 & 67,5 bB 78,3 bA & 93,3 abA 93,3 aA & 96,3 aA $95,3 \mathrm{bcA}$ & 96,8 aA 94,3 bB & 99,0 aA 97,8 abA \\
\hline 2. GR-142 + DMA 8062 & $25+670$ & 64,5 bA 46,3 cB & 85,3 bA 71,5 bB & 98,5 aA $93,3 \mathrm{cB}$ & 97,0 aA $95,8 \mathrm{abA}$ & 99,5 aA 97,5 bB \\
\hline 3. GR-142? & 50 & 96,3 aA 90,3 aB & 99,5 aA 99,5 aA & 100,0 aA 100,0 aA & 99,5 aA 98,5 aA & 100,0 aA 99,5 abA \\
\hline 4. GR-142 + DMA 8062 & $50+670$ & 91,3 aA 76,5 bB & 99,8 aA 95,3 aA & 100,0 aA 99,3 abA & 100,0 aA 97,8 abA & 100,0 aA 99,8 abA \\
\hline 5. GR-142 & 62 & 96,8 aA 87,0 aB & 99,5 aA 99,8 aA & 100,0 aA 99,5 aA & 100,0 aA 98,5 aA & 100,0 aA 100,0 aA \\
\hline 6. GR-142 + DMA 8062 & $62+670$ & 96,8 aA 78,3 bB & 99,8 aA 96,0 aA & 100,0 aA 98,5 abA & 100,0 aA 97,8 abA & 100,0 aA 99,5 abA \\
\hline 7. Select ${ }^{\frac{3}{4}}$ & 84 & 98,5 aA 91,3 aB & 100,0 aA 99,3 aA & 100,0 aA 99,8 aA & 100,0 aA 99,5 aA & 100,0 aA 99,5 abA \\
\hline 8. Select + DMA $806^{3}$ & $84+670$ & 99,5 aA 87,0 aB & 100,0 aA 95,0 aA & 100,0 aA 97,8 abA & 99,5 aA 98,0 abA & 100,0 aA 99,8 abA \\
\hline 9. Testemunha sem aplicação & - & $0,0 \quad$ c $\quad 0,0 \quad$ d & $0,0 \mathrm{c}$ & $0,0 \mathrm{~d}$ & $0,0 \mathrm{~b}$ & 0,0 b $\quad 0,0$ \\
\hline F Tratamento & & $580,41^{*}$ & $329,43^{*}$ & $2832,80^{*}$ & $2962,04^{*}$ & $7666,71^{*}$ \\
\hline FEstádio & & $98,73^{*}$ & $6,55^{*}$ & $9,60^{*}$ & $12,44^{*}$ & $5,34^{*}$ \\
\hline F Tratamento $x$ Estádio & & $13,42^{*}$ & $1,61^{\mathrm{NS}}$ & $1,80^{\mathrm{NS}}$ & $0,48 \mathrm{NS}^{-1}$ & $0,74 \mathrm{NS}^{-1}$ \\
\hline CV $(\%)$ & & 4,84 & 5,97 & 2,00 & 1,95 & 1,21 \\
\hline DMS Tratamento & & 8,28 & 11,60 & 4,00 & 3,90 & $2,45^{-}$ \\
\hline DMS Estádio & & 5,13 & 7,19 & 2,48 & 2,42 & 1,52 \\
\hline
\end{tabular}

${ }^{1 /} \mathrm{EF}=$ Estádio de desenvolvimento das plantas de milho com 5 ou 7 folhas totalmente expandidas (V5 ou V7); ${ }^{2 /}$ Utilizou-se 1,0 L pc ha ${ }^{-1}$ de Joint $\mathrm{Oil}^{\circledR}$ (Adjuvante do grupo dos hidrocarbonetos alifáticos); ${ }^{\circledR} /{\text { Utilizou-se } 1,0 \mathrm{~L} \mathrm{pc} \mathrm{ha}^{-1} \text { de Lanzar }}^{\circledR}$ (Adjuvante do grupo dos alquil ester etoxilado do ácido fosfórico); - DAA = dias após aplicação; - Médias na mesma coluna seguidas da mesma letra não diferem entre si, a 5\% de probabilidade pelo teste de Tukey. ${ }^{*} \mathrm{p} \leq 0,05{ }^{\mathrm{NS}}=$ não significativo.

Outra similaridade constatada foi $\mathrm{o}$ controle altamente eficiente de todos os híbridos de milho $\mathrm{RR}$ nos estádios $\mathrm{V}_{5}$ e $\mathrm{V}_{7}$, respectivamente, a partir dos 7 e 14 DAA ( $\leq$ $91,5 \%$ e $97,5 \%$ ) com 92,6 e $115,0 \mathrm{~g} \mathrm{ha}^{-1}$ de GR-142 isolado, não diferindo significativamente do tratamento com Select ${ }^{\circledR}$. Esses resultados corroboram com os obtidos por Soares et al. (2010), onde utilizando os herbicidas clethodim $\left(84 ; 108 \mathrm{~g} \mathrm{ha}^{-1}\right)$, sethoxydim $\left(184 ; 230 \mathrm{~g} \mathrm{ha}^{-1}\right)$, tepraloxydim $\left(80 ; 100 \mathrm{~g} \mathrm{ha}^{-1}\right)$, clethodim+fenoxaprop-p-ethyl $\left(40+40 ; 50+50 \mathrm{~g} \mathrm{ha}^{-1}\right)$, fluazifop-p-butyl (125; $\left.188 \mathrm{~g} \mathrm{ha}^{-1}\right)$ e haloxyfop-methyl (50; $\left.62 \mathrm{~g} \mathrm{ha}^{-1}\right)$ em pós-emergência da soja RR, constataram alta eficiência de controle variando de $92 \%$ a $100 \%$ aos 28 DAA para os híbridos DKB390RR2 e AG7088RR2, em estádio $\mathrm{V}_{5} / \mathrm{V}_{6}$. De forma semelhante, Bianchi (2009) e Schneider et al. (2011) também verificaram controle de milho RR voluntário utilizando clethodim $\left(84,0 \mathrm{~g} \mathrm{ha}^{-1}\right)$ e fluazifop-p-butyl $\left(187,5 \mathrm{~g} \mathrm{ha}^{-1}\right)$ em estádio $\mathrm{V}_{4}$, e haloxyfopmethyl $\left(62,4\right.$ a $\left.155,9 \mathrm{~g} \mathrm{ha}^{-1}\right)$, clethodim $(108,0$ $\left.\mathrm{g} \mathrm{ha}^{-1}\right)$, quizalofop $\left(48,0 \mathrm{~g} \mathrm{ha}^{-1}\right)$ e sethoxydim $\left(230,0\right.$ g i.a. ha $\left.^{-1}\right)$, em estádio $V_{6} / V_{8}$, respectivamente.

Em termos de associação, Soltani et al. (2006) relataram que misturas em tanque de glyphosate e graminicidas é dependente da dose utilizada, sendo que baixas doses de clethodim, fenoxaprop-p-ethyl e fluazifop-pbutyl resultaram em controle inadequado ( $<$ $80 \%$ ) de milho voluntário $\mathrm{RR}^{\circledR}$. Já Ferreira Neto et al. (2012), constataram controle eficiente de milho voluntário $R R$ em estádio $V_{4}$ e $\mathrm{V}_{7}$, variando entre 88 e $92 \%$ aos 14 DAA, utilizando glyphosate (Roundup Ready ${ }^{\circledR} 2,0 \mathrm{~L}$ pc $\mathrm{ha}^{-1}$ ) em mistura em tanque com os graminicidas Poquer $^{\circledR}$ (clethodim $96 \mathrm{~g} \mathrm{ha}^{-1}$ ), Poast $^{\circledR}$ (sethoxidim $184 \mathrm{~g} \mathrm{ha}^{-1}$ ), Fusilade $250^{\circledR}$ (fluazifop-p-butilico 187,5 $\mathrm{g} \mathrm{ha}^{-1}$ ), Aramo $200^{\circledR}$ (teprloxidim $100 \mathrm{~g} \mathrm{ha}^{-1}$ ), Podium $\mathrm{S}^{\circledR}$ (fenoxaprop-p-etílico+clethodim $30+30 \mathrm{~g} \mathrm{ha}^{-1}$ ), Targa $^{\circledR}$ (quizalofop-p-etílico $40 \mathrm{~g} \mathrm{ha}^{-1}$ ) e Verdict $\mathrm{R}^{\circledR}$ (haloxifop-p-etil $125 \mathrm{~g} \mathrm{ha}^{-1}$ ), na cultura da soja $\mathrm{RR}^{\circledR}$. 

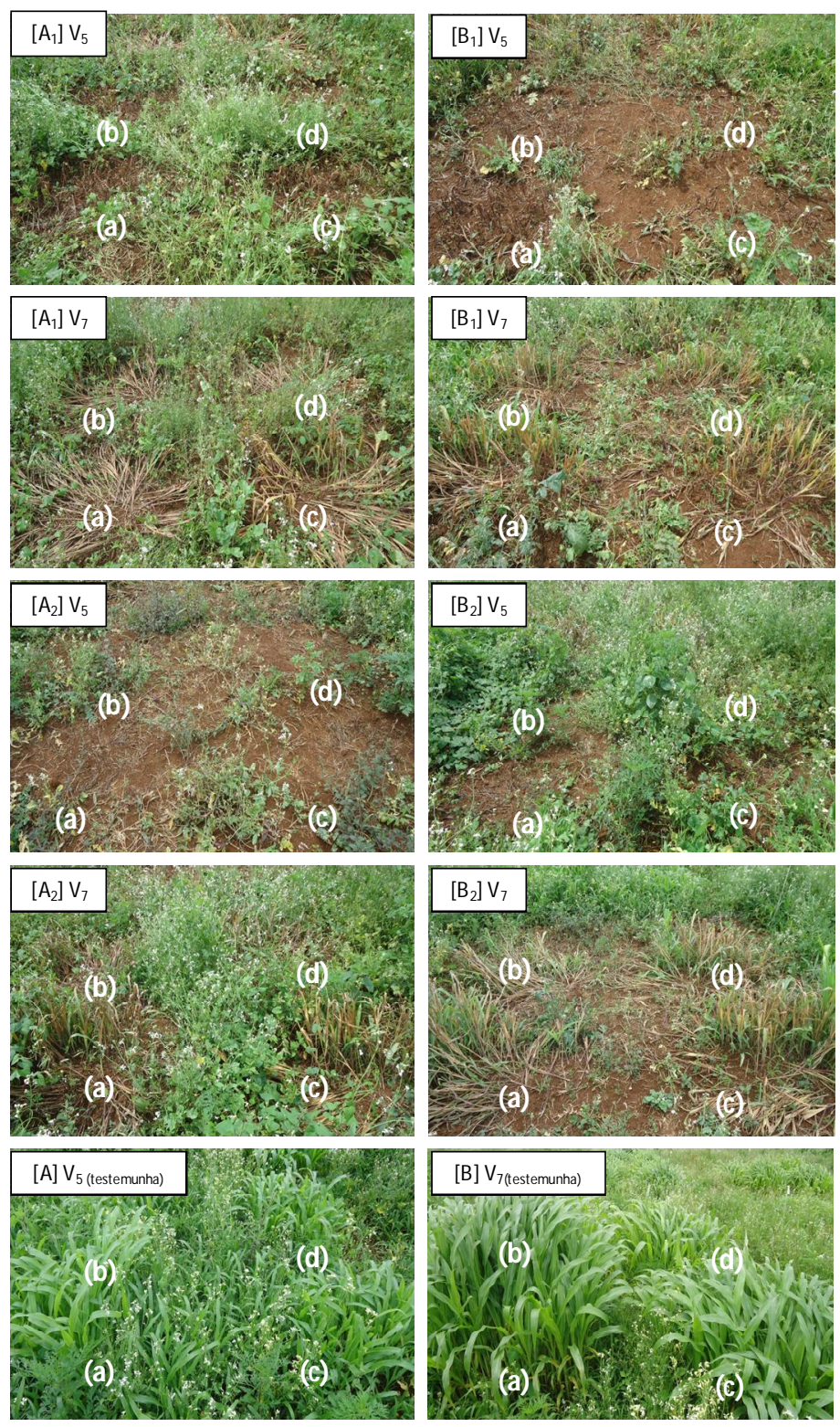

Figura 2. Ilustração do controle de milho RR voluntário DKB 240 PRO 2 (a), AG 8025 RR2 (b), Pioneer 30F 53HR (c) e AG 9045 RR2 (d) aos 14 dias após aplicação (DAA) em pós-emergência dos tratamentos herbicida GF-142 $\left(62 \mathrm{~g} \mathrm{ha}^{-1}\right)\left[\mathrm{A}_{1}\right]$, GF-142 + DMA $806 \mathrm{BR}^{\circledR}\left(62+670 \mathrm{~g} \mathrm{ha}^{-1}\right)$ $\left[\mathrm{B}_{1}\right]$, Select $^{\circledR}\left(84 \mathrm{~g} \mathrm{ha}^{-1}\right)\left[\mathrm{A}_{2}\right]$ e Select $^{\circledR}+\mathrm{DMA}^{806 \mathrm{BR}^{\circledR}}\left(84+670 \mathrm{~g} \mathrm{ha}^{-1}\right)\left[\mathrm{B}_{2}\right]$ e testemunhas em estádios $V_{5}$ e V 7 . Guarapuava - PR, 2012/2013.

De forma geral, todos os tratamentos com GR-142 (25; 50 e $\left.62 \mathrm{~g} \mathrm{ha}^{-1}\right)$, isolados e/ou em mistura com DMA $806 \mathrm{BR}^{\circledR}\left(670 \mathrm{~g} \mathrm{ha}^{-1}\right)$, foram altamente eficientes no controle dos híbridos DKB 240 PRO2, AG 8025 RR2 e AG 9045 RR2 a partir dos 21 DAA, e no controle do híbrido Pionner 30F 53HR, a partir dos 28
DAA, ambos nos estádios $\mathrm{V}_{5}$ e $\mathrm{V}_{7}$. Entretanto, o controle inicial lento e insuficiente da dose de $25 \mathrm{~g} \mathrm{ha}^{-1}$ de GR-142 para todos os híbridos, evidenciou a necessidade de complemento da sua concentração para melhorar a velocidade de ação, principalmente quando utilizado em mistura com DMA $806 \mathrm{BR}^{\circledR}$. 
Tabela 5. Porcentagens de controle de milho RR híbrido voluntário Pioneer 30F 53HR aos 7, 14, 21, 28 e 42 dias após aplicação (DAA) em pós-emergência do herbicida GF-142 [haloxyfopmethyl (R)], em estádios de desenvolvimento $V_{5}$ e $V_{7}$. Guarapuava - PR, 2012/2013.

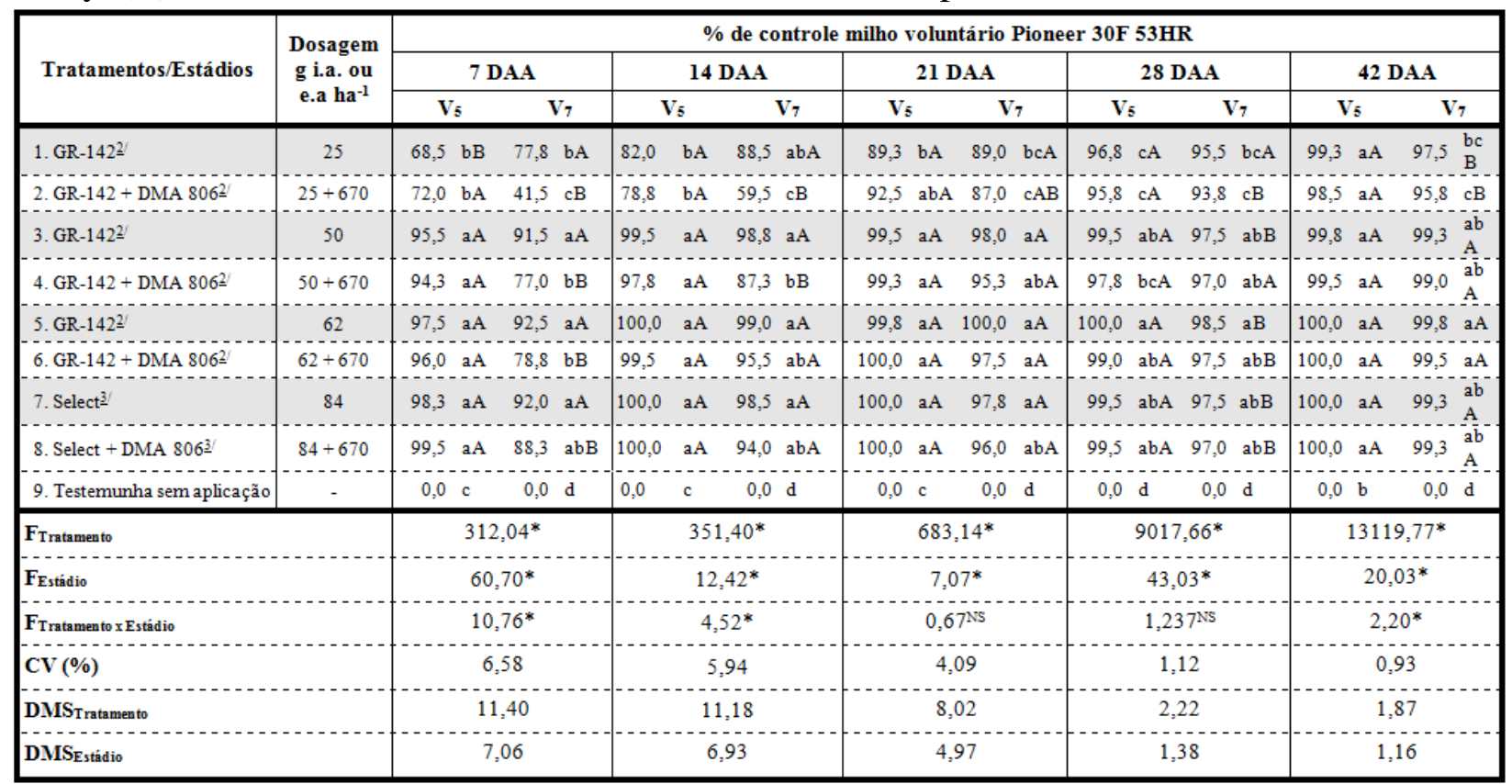

${ }^{1 /} \mathrm{EF}=$ Estádio de desenvolvimento das plantas de milho com 5 ou 7 folhas totalmente expandidas (V5 ou V7); ${ }^{2 /}$ Utilizou-se $1,0 \mathrm{~L}$ pc ha ${ }^{-1}$ de Joint $\mathrm{Oil}^{\circledR}$ (Adjuvante do grupo dos hidrocarbonetos alifáticos); ${ }^{3 /}$ Utilizou-se $1,0 \mathrm{~L} \mathrm{pc} \mathrm{ha}{ }^{-1}$ de Lanzar ${ }^{\circledR}$ (Adjuvante do grupo dos alquil ester etoxilado do ácido fosfórico); - DAA = dias após aplicação; - Médias na mesma coluna seguidas da mesma letra não diferem entre si, a 5\% de probabilidade pelo teste de Tukey. ${ }^{*} \mathrm{p} \leq 0,05{ }^{\text {NS }}=$ não significativo.

Tabela 6. Porcentagens de controle de milho RR híbrido voluntário AG 9045 RR2 aos 7, 14, 21, 28 e 42 dias após aplicação (DAA) em pós-emergência do herbicida GF-142 [haloxyfop-methyl (R)], em estádios de desenvolvimento $\mathrm{V}_{5}$ e $\mathrm{V}_{7}$. Guarapuava - PR, 2012/2013.

\begin{tabular}{|c|c|c|c|c|c|c|c|c|c|c|c|c|c|c|c|c|}
\hline \multirow{3}{*}{ Tratamentos/Estádios } & \multirow{3}{*}{$\begin{array}{c}\text { Dosagem } \\
\text { g i.a. ou } \\
\text { e.a ha }{ }^{-1}\end{array}$} & \multicolumn{15}{|c|}{ \% de controle milho voluntário AG 9045 RR2 } \\
\hline & & \multicolumn{3}{|c|}{7 DAA } & \multicolumn{3}{|c|}{14 DAA } & \multicolumn{3}{|c|}{21 DAA } & \multicolumn{3}{|c|}{28 DAA } & \multicolumn{3}{|c|}{42 DAA } \\
\hline & & $V_{5}$ & & $\mathrm{~V}_{7}$ & $V_{5}$ & $\mathrm{~V}_{7}$ & & $V_{5}$ & $\mathrm{~V}_{7}$ & & $V_{5}$ & & $\mathrm{~V}_{7}$ & $\mathbf{V}_{5}$ & & $V_{7}$ \\
\hline 1. GR-142 & 25 & 66,3 b & bB & 79,0 dA & $76,5 \mathrm{~b}$ & 88,3 & & $91,5 \mathrm{r}$ & 95,8 & & 96,8 & $\mathrm{cA}$ & $96,5 \mathrm{bA}$ & 98,8 & 98,0 & $0 \mathrm{a}$ \\
\hline 2. GR-142+ DMA 8062 & $25+670$ & 59,5 b & bA & $47,8 \mathrm{eB}$ & $62,0 \mathrm{c}$ & 64,5 & b & 93,8 & 95,3 & & 96,8 & cA & $96,3 \mathrm{bA}$ & 98,5 & 98,8 & 8 a \\
\hline 3. GR-142 & 50 & 96,0 a & aA & 92,8 aA & 99,3 a & 99,5 & & $99,8=$ & 99,3 & $\mathrm{ab}$ & 100,0 & aA & 98,3 abB & 100,0 & 99,5 & 5 a \\
\hline 4. GR-142 + DMA 806? & $50+670$ & 93,5 a. & aA & $82,0 \mathrm{cdB}$ & $96,5=$ & 88,3 & a & $99,0=$ & 98,5 & & 97,8 & $B$ bcA & $98,0 \mathrm{abA}$ & 100,0 & 99,8 & $8 \mathrm{a}$ \\
\hline 5. GR-142? & 62. & 95,8 a & aA & $92,5 \mathrm{abA}$ & $97,5 \mathrm{a}$ & 99,8 & a & $100,0=$ & 100,0 & & 99,0 & $\mathrm{abA}$ & 99,0 aA & 99,8 & 99,8 & 8 a \\
\hline 6. GR-142 + DMA 8062 & $62+670$ & 94,5 a & aA & 83,8 bcdB & $98,8=$ & 95,3 & & $100,0=$ & 98,3 & & 99,0 & $a b A$ & 97,8 abA & 100,0 & 99,5 & 5 a \\
\hline 7. Select $\mathrm{z}^{3 /}$ & 84 & 99,3 at & aA & 94,5 aA & $100,0=$ & 98,5 & $\begin{array}{c}a \\
-.\end{array}$ & $100,0=$ & 99,3 & $a b$ & 99,5 & $\mathrm{abA}$ & 98,0 abB & 100,0 & 99,8 & 8 a \\
\hline 8. Select + DMA 8063 & $84+670$ & 100,0 a & aA & 90,0 abcB & 100,0 a & 95,3 & & 100,0 & 97,5 & $\mathrm{ab}$ & 100,0 & aA & 97,8 abB & 100,0 & 99,5 & 5 a \\
\hline 9. Testemunha sem aplicação & - & $0,0 \mathrm{c}$ & c & $0,0 \mathrm{f}$ & $0,0 \mathrm{~d}$ & 0,0 & & 0,0 & 0,0 & & & $\mathrm{~d}$ & 0,0 & 0,0 & 0,0 & $0 \mathrm{~b}$ \\
\hline \multicolumn{2}{|l|}{$F_{\text {Tratamento }}$} & \multicolumn{3}{|c|}{$534,81^{*}$} & \multicolumn{3}{|c|}{$226,48^{*}$} & \multicolumn{3}{|c|}{$2288,98^{*}$} & \multicolumn{3}{|c|}{$10809,30 *$} & \multicolumn{3}{|c|}{$10626,15^{*}$} \\
\hline \multicolumn{2}{|l|}{ Festadio } & \multicolumn{3}{|c|}{$26,63^{*}$} & \multicolumn{3}{|c|}{$0,01^{\mathrm{NS}}$} & \multicolumn{3}{|c|}{$0,004 \mathrm{Ns}^{-1}$} & \multicolumn{3}{|c|}{$14,73^{*}$} & \multicolumn{3}{|c|}{$1,68 \mathrm{Ns}^{-1}$} \\
\hline \multicolumn{2}{|l|}{ F Tratamento x Estádio } & \multicolumn{3}{|c|}{$8,15^{*}$} & \multicolumn{3}{|c|}{$1,69 \mathrm{NS}$} & \multicolumn{3}{|c|}{$2,05 \mathrm{Ns}^{-}$} & \multicolumn{3}{|c|}{$2,04 \mathrm{Ns}^{-1}$} & \multicolumn{3}{|c|}{$0,24 \mathrm{Ns}^{-1}$} \\
\hline \multicolumn{2}{|l|}{ CV $(\%)$} & \multicolumn{3}{|c|}{5,11} & \multicolumn{3}{|c|}{7,56} & \multicolumn{3}{|c|}{2,22} & \multicolumn{3}{|c|}{4,43} & \multicolumn{2}{|r|}{1,03} & \\
\hline DMS ${ }_{\text {Tratamento }}$ & & & 8,89 & & & 05 & & & 43 & & & 8,8 & & & 2,08 & \\
\hline DMSEstadio & & & 5,51 & & & 34 & & &, $75^{-1}$ & & & 1,2 & & & $2,55^{-}$ & \\
\hline
\end{tabular}

${ }^{1 /} \mathrm{EF}=$ Estádio de desenvolvimento das plantas de milho com 5 ou 7 folhas totalmente expandidas (V5 ou V7); ${ }^{2 /}$ Utilizou-se 1,0 L pc ha ${ }^{-1}$ de Joint $\mathrm{Oil}^{\circledR}$ (Adjuvante do grupo dos hidrocarbonetos alifáticos); ${ }^{3 /}$ Utilizou-se $1,0 \mathrm{~L} \mathrm{pc} \mathrm{ha}^{-1}$ de Lanzar ${ }^{\circledR}$ (Adjuvante do grupo dos alquil ester etoxilado do ácido fosfórico); - DAA = dias após aplicação; - Médias na mesma coluna seguidas da mesma letra não diferem entre si, a 5\% de probabilidade pelo teste de Tukey. ${ }^{*} \mathrm{p} \leq 0,05{ }^{\mathrm{NS}}=$ não significativo. 


\section{Conclusões}

O herbicida haloxyfop-R (GR-142), nas doses de 25; 50 e $62 \mathrm{~g} \mathrm{ha}^{-1}$, assim como o clethodim $\left(\right.$ Select $\left.^{\circledR}\right)$ a $84 \mathrm{~g} \mathrm{ha}^{-1}$, isolados ou em mistura em tanque com DMA $806 \mathrm{BR}^{\circledR}(670 \mathrm{~g}$ $\mathrm{ha}^{-1}$ ) foram eficientes no controle em pósemergência dos híbridos de milho DKB 240 PRO2, AG 8025 RR2, AG 9045 RR2 e Pionner 30F 53HR, nos estádios de desenvolvimento $\mathrm{V}_{5}$ e $\mathrm{V}_{7}$. O haloxyfop-R (GR-142), nas doses de 50 e $62 \mathrm{~g} \mathrm{ha}^{-1}$, isolado ou em mistura com DMA $806 \mathrm{BR}^{\circledR} \quad\left(670 \mathrm{~g} \mathrm{ha}^{-1}\right)$, promoveu controle eficiente e precoce dos híbridos a partir dos 7 DAA no estádio $\mathrm{V}_{5}$, e com eficiência mais lenta, observada a partir dos 14 e 21 DAA para o estádio $V_{7}$.

\section{Referências}

BIANCHI, M.A. Avaliação da eficiência e seletividade de clethodin sobre milho voluntário (Zea mays L.) na cultura da soja. In: Resultados de pesquisa: controle de plantas daninhas 1993 a 2008. Cruz Alta: Fundacep Fecotrigo, 2009. p.178-180.

DAVIS, V.M.; MARQUARDT, P.T.; JOHNSON, W.J. Volunteer corn in northern Indiana soybean correlates to glyphosateresistant corn adoption. Crop Management, 2008. doi: 10.1094/CM-2008-0721-01-BR. <http://www.plantmanagementnetwork.org/pu b/ cm/brief/2008/volunteer/> (Accesso em: 20/09/2013).

DEEN, W.; HAMILL, A; SHROPSHIRE, C.; SOLTANI, N.; SIKKEMA, P.H. Control of volunteer glyphosate-resistant corn (Zea mays) in glyphosate resistant soybean (Glycine max). Weed Technology, v.20, n.1, p.261-266, 2006.

EMPRESA BRASILEIRA DE PESQUISA AGROPECUÁRIA - EMBRAPA SOLOS. Centro Nacional de Pesquisa de Solos. Sistema brasileiro de classificação de solos. 2. ed. Brasília, 2006. 306p.
FERREIRA NETO, A.; DALMAZZO; C.H.; MONTEZUMA, M.C.; PEREIRA, B.F. PEREIRA, D.M. Controle de milho Roundup Ready $^{\circledR}$ como planta voluntária em soja Roundup Ready ${ }^{\circledR}$. In: XXVIII CONGRESSO BRASILEIRO DA CIÊNCIA DAS PLANTAS DANINHAS. Resumos expandidos... Ribeirão Preto: SBCPD, 2012. p.315-319. CD-ROOM.

MARQUARDT, P.T.; TERRY, R.M.; KRUPKE, C.H.; JOHNSON, W.G. Competitive effects of volunteer corn on hybrid corn growth and yield. Weed Science, v.60, n.4, p.537-541, 2012.

PEEL, M.C.; FINLAYSON, B.L.; MCMAHON, T.A. Updated world map of the Köppen-Geiger climate classification. Hydrology and Earth System Sciences, v.11, n.5, p.1633-1644, 2007.

RODRIGUES, B.N.; ALMEIDA, F.S. Guia de herbicidas. 6.ed. Londrina: IAPAR, 2011 697p.

ROMAN, E.S.; BERCKIE, H.; VARGAS, L.; HALL, L.; RIZZARDI, M.A.; WOLF, T.M. Como funcionam os herbicidas: da biologia à aplicação. Passo Fundo: Editora Berthier, 2007. 160p.

SBCPD - Sociedade Brasileira da Ciência das Plantas Daninhas. Procedimentos para instalação, avaliação e análise de experimentos com herbicidas. $1^{\text {a }}$ ed. Londrina: SBCPD, 1995. 42p.

SCHNEIDER, T.; ROCKENBACH, A.C.; BIANCHI, M.A. Controle de milho resistente ao glifosato com herbicidas inibidores da enzima Acetil Coenzima A Carboxilase. In: XVI SEMINÁRIO INTERINSTITUCIONAL DE ENSINO, PESQUISA E EXTENSÃO. Anais... Cruz Alta: UNICRUZ, 2011. CDROOM.

SOARES, D.J.; VERTUAN, H.V.; MOTOMIYA, W.R.; MACEDO, F.B.; DOURADO, P.M.; OLIVEIRA, W.S.; LÓPEZ-OVEJERO, R.F. Controle de plantas 
voluntárias de milho geneticamente modificado tolerante ao glifosato na cultura da soja. In: XXVII CONGRESSO BRASILEIRO DA CIENCIA DAS PLANTAS DANINHAS. Resumos expandidos... Ribeirão Preto: SBCPD, 2010. p.1513-1516. CD-ROOM.

SOLTANI, N.; SHROPSHIRE, C.; SIKKEMA, P.H. Control of volunteer glyphosate-tolerant maize (Zea mays) in glyphosate-tolerant soybean (Glycine max). Crop Protection, v.25, n.2, p.178-181, 2006.

TREZZI, M.M; MATEI, D.; VIDAL, R.A.; KRUSE, N.D.; GUSTMAM, M.S.; VIOLA, R.; MACHADO, A.; SILVA, H.L. Antagonismo das associações de clodinafoppropargyl com metsulfuron methyl e 2,4-D no controle de azevém (Lolium multiflorum). Planta Daninha, v.25, n.4, p.839-847, 2007.

VIDAL, R.A.; MEROTTO JÚNIOR, A. Herbicidologia. Porto Alegre: Evangraf, 2001. $152 \mathrm{p}$.

YOUNG, B.G.; HART, S.E. Control of volunteer sethoxydim-resistant corn (Zea mays) in soybean (Glycine max). Weed Technology, v.11, n.4, p.69-655, 1997. 